Article

\title{
Hierarchical Self-Assembled Structures from Diblock Copolymer Mixtures by Competitive Hydrogen Bonding Strength
}

\author{
Tzu-Chun Tseng ${ }^{1}$ and Shiao-Wei Kuo ${ }^{1,2, *}$ \\ 1 Department of Materials and Optoelectronic Science, Center for Functional Polymers \\ and Supramolecular Materials, National Sun Yat-Sen University, Kaohsiung 80424, Taiwan; \\ mix50832@gmail.com \\ 2 Department of Medicinal and Applied Chemistry, Kaohsiung Medical University, Kaohsiung 80424, Taiwan \\ * Correspondence: kuosw@faculty.nsysu.edu.tw; Tel./Fax: +886-752-540-99
}

Academic Editor: Zibiao Li

Received: 19 August 2018; Accepted: 31 August 2018; Published: 3 September 2018

\begin{abstract}
In this work we prepared poly(styrene- $b$-vinylphenol) (PS- $b$-PVPh) by sequential anionic living polymerization and poly(ethylene oxide- $b$-4-vinylpyridine) (PEO- $b$-P4VP) by reversible addition fragmentation chain transfer polymerization (RAFT) by using poly(ethylene oxide) 4-cyano-4-(phenylcarbonothioylthio)pentanoate (PEO-SC(S)Ph) as a macroinitiator with two hydrogen bonded acceptor groups. When blending with disordered PEO-b-P4VP diblock copolymer, we found the order-order self-assembled structure transition from lamellar structure for pure PS- $b$-PVPh to cylindrical, worm-like, and finally to PEO crystalline lamellar structures. Taking the advantage of the $\Delta K$ effect from competitive hydrogen bonding strengths between $\mathrm{PVPh} / \mathrm{P} 4 \mathrm{VP}$ and $\mathrm{PVPh} / \mathrm{PEO}$ domains, it could form the hierarchical self-assembled morphologies such as core-shell cylindrical nanostructure.
\end{abstract}

Keywords: self-assembly; hydrogen bonding; block copolymers; living polymerization

\section{Introduction}

Diblock copolymers can display different self-assembly behaviors, including lamellar, gyroid, cylinder, and spherical nanostructures, which possess several potential applications in drug delivery, photonic crystals, and nanotechnology [1-4]. However, mediating the different molecular weights of each block segment to control the volume fraction by using living polymerization methods may be time-consuming and difficult and thus blending the homopolymer or another block copolymer through intermolecular hydrogen bonding interactions has received much interest recently [5-7]. For diblock copolymer/homopolymer $(\mathrm{A}-b-\mathrm{B} / \mathrm{C})$ blends with hydrogen bonding interactions, there are four different situations that have been proposed based on experimental and theoretical results [8-25]. However, only the typical self-assembly nanostructures as diblock copolymers were observed for A- $b$ - $\mathrm{B} / \mathrm{C}$ blends including PS- $b$-PVPh blending with poly(methyl methacrylate) (PMMA), polycaprolactone (PCL), PEO, P2VP, and P4VP homopolymers; PS- $b$-P2VP, and PCL- $b$-P4VP diblock copolymers blending PVPh homopolymer; PVPh- $b$-PCL or PVPh- $b$-PMMA blending with PVP homopolymer [8-25].

Furthermore, extending the $\mathrm{A}-b-\mathrm{B} / \mathrm{C}$ mixtures to $\mathrm{A}-b-\mathrm{B} / \mathrm{C}-b-\mathrm{D}$ diblock copolymer mixtures as similar triblock copolymer has also received much interest recently since they provide a simpler approach for the formation of hierarchical self-assembled structures such as three lamellae or core-shell cylinder structures [26-38]. In general, the B and C blocks would be miscible through hydrogen bonding interactions, but the $\mathrm{A}$ and $\mathrm{D}$ blocks are immiscible with each other. For example, Matsushita and we have found three phase nanostructures either in bulk or in solution state for 
PS- $b$-PVPh blended with P4VP- $b$-PCL, P4VP-b-PMMA, and P4VP-b-PI [26-29]. In these cases, these block segments are immiscible. To the best of our knowledge, there are few studies that investigate immiscible A-b-B with disordered C- $b$-D block copolymers to provide hierarchical self-assembled structures. In our previous work [39], we proposed immiscible PS- $b$-P4VP blending with miscible disordered PVPh- $b$-PMMA to form hierarchical self-assembled structures such as three lamellar or core-shell cylinder nanostructures. In this work, we propose another immiscible PS- $b$-PVPh diblock copolymer blend with disordered PEO- $b$-P4VP diblock copolymer, which is also mediated by using competitive hydrogen bonding strength to form the hierarchical self-assembled structures.

\section{Results and Discussion}

\subsection{The Preparation of PS-b-PVPh and PEO-b-P4VP Diblock Copolymers}

In previous studies $[11,13,40]$ we have proposed the preparation of PS- $b$-PVPh diblock copolymers using anionic polymerization and then performing a hydrolytic reaction to remove tert-butyl ether units from poly(tert-butoxystyrene) (PtBuOS, Scheme 1a). We observed the formation of phenolic $\mathrm{OH}$ units for PS-b-PVPh diblock copolymer based on the complete removal of the protective tert-butyl groups based on FTIR, NMR and GPC analyses. Firstly, the PS- $b$-PVPh spectrum with a broad band at $3360 \mathrm{~cm}^{-1}$ and a sharp absorption at $3530 \mathrm{~cm}^{-1}$, represents the self-association and free $\mathrm{OH}$ groups after deprotection as shown in Figure $1 \mathrm{~A}(\mathrm{~b})$. Secondly, the signal at $1.30 \mathrm{ppm}$ in the ${ }^{1} \mathrm{H}-\mathrm{NMR}$ (Figure 1B(a)) and $78.0 \mathrm{ppm}$ in the ${ }^{13} \mathrm{C}-\mathrm{NMR}$ (Figure 1C(a)) spectra of PS-b-PtBuOS diblock copolymer both correspond to the tert-butyl ether groups and both signals disappeared after the hydrolysis reaction, while a signal at $9.0 \mathrm{ppm}$ corresponding to $\mathrm{OH}$ units of $\mathrm{PVPh}$ block appeared (Figure $1 \mathrm{~B}(\mathrm{~b})$ ). In addition, the signal of the $\mathrm{Cf}-\mathrm{OH}$ unit was shifted from $153.6 \mathrm{ppm}$ (Figure $1 \mathrm{C}(\mathrm{a})$ ) to $155.8 \mathrm{ppm}$ (Figure $1 \mathrm{C}(\mathrm{b})$ ), also indicating the complete hydrolysis. Finally, the GPC trace of PS- $b$-PVPh displays a higher molecular weight shift compared with PS- $b$-PtBuOS, probably due to the polymer-stationary phase interaction because of the hydrogen bonding interaction, as shown in Figure 1D. The number average molecular weight $(\mathrm{Mn})$ and polydispersity of $\mathrm{PS}_{460}-b-\mathrm{PVPh}_{158}$ were calculated as 63,000 g/mole and 1.04 based on Figure 1D(a).

(a)

sec-BuLi +<smiles>CC(C)CC(C)c1ccccc1</smiles>

$\mathrm{MeOH}$

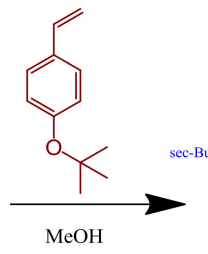<smiles>CCCc1cccc2c(OC(C)(C)C)ccc(C(C)C)c12</smiles>

PS- $b$-PtBuOS

(b)

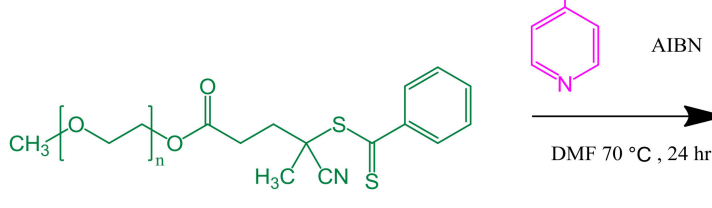

PEO-RAFT

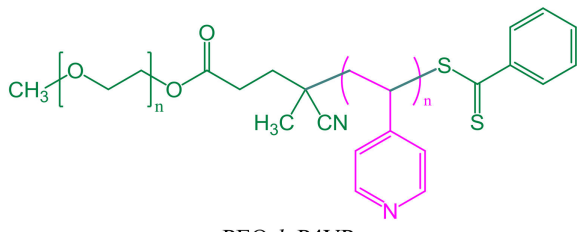

PEO- $b$-P4VP

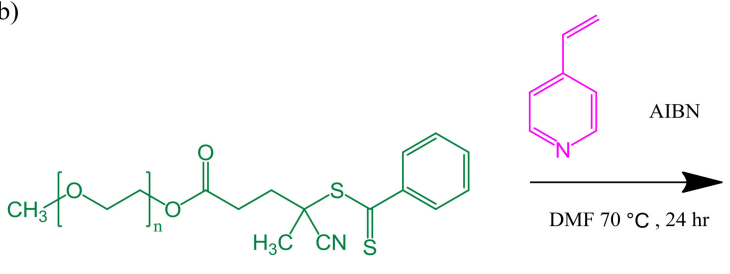

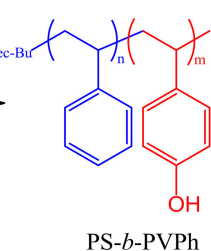

PS- $b$-PVPh

Scheme 1. Synthesis of (a) PS- $b$-PVPh by sequential anionic living polymerization and (b) PEO- $b$-P4VP by RAFT polymerization. 
We also synthesized the PEO-b-P4VP diblock copolymer by RAFT polymerization as shown in Scheme 1b. Firstly, the spectrum of the PEO-RAFT macro-initiator displays a C-O-C stretching band at $1110 \mathrm{~cm}^{-1}$ (Figure 2A(a)) and the PEO-b-P4VP diblock copolymer features extra pyridine units at 993, 1598 and $3023 \mathrm{~cm}^{-1}$ as shown in Figure 2A(b). Furthermore, the $\mathrm{CH}_{2}$ unit of PEO segment was located at $3.50 \mathrm{ppm}$ in the ${ }^{1} \mathrm{H}-\mathrm{NMR}$ spectrum and the other peak assignments of PEO-RAFT macro-initiator are displayed in Figure 2B(a).

(A)

(B)

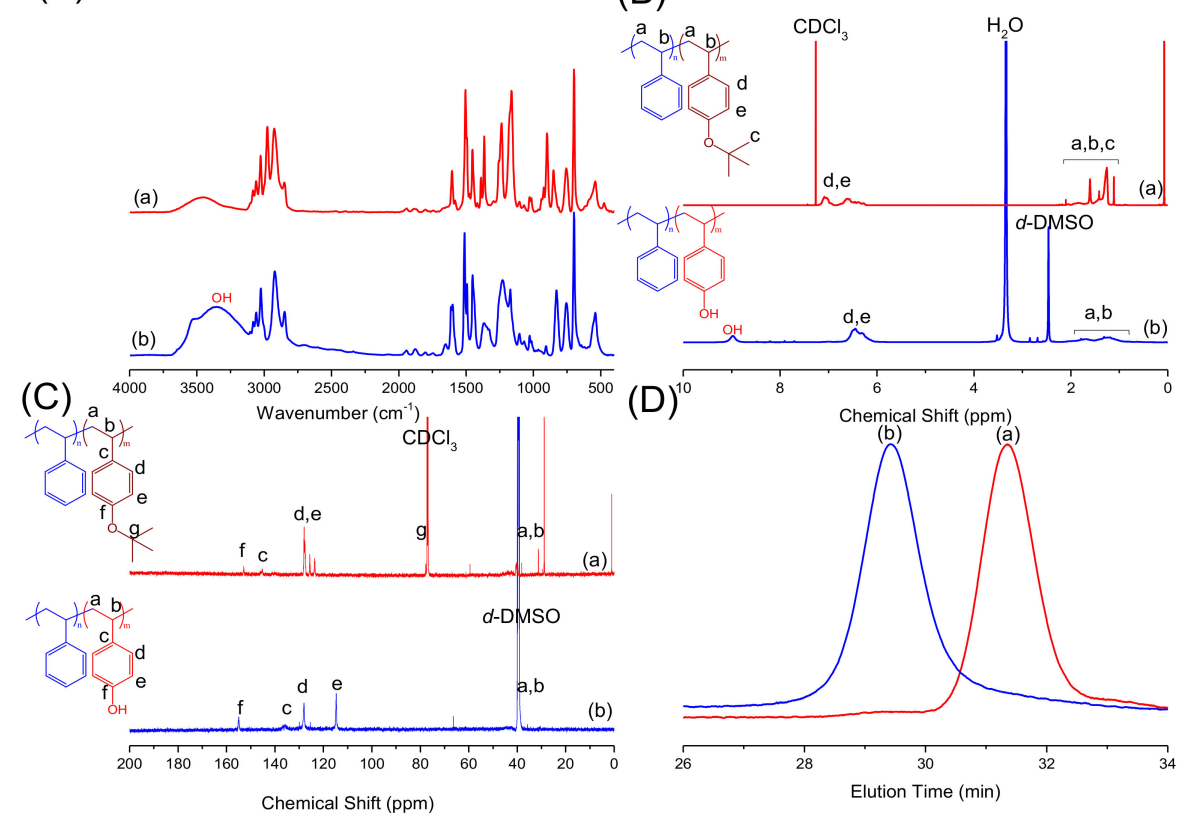

Figure 1. (A) FTIR, (B) ${ }^{1} \mathrm{H}-,\left(\right.$ C) ${ }^{13} \mathrm{C}-\mathrm{NMR}$, and (D) GPC analyses of (a) PS- $b$-PtBuOS and (b) PS- $b$-PVPh.

(A)

(B)

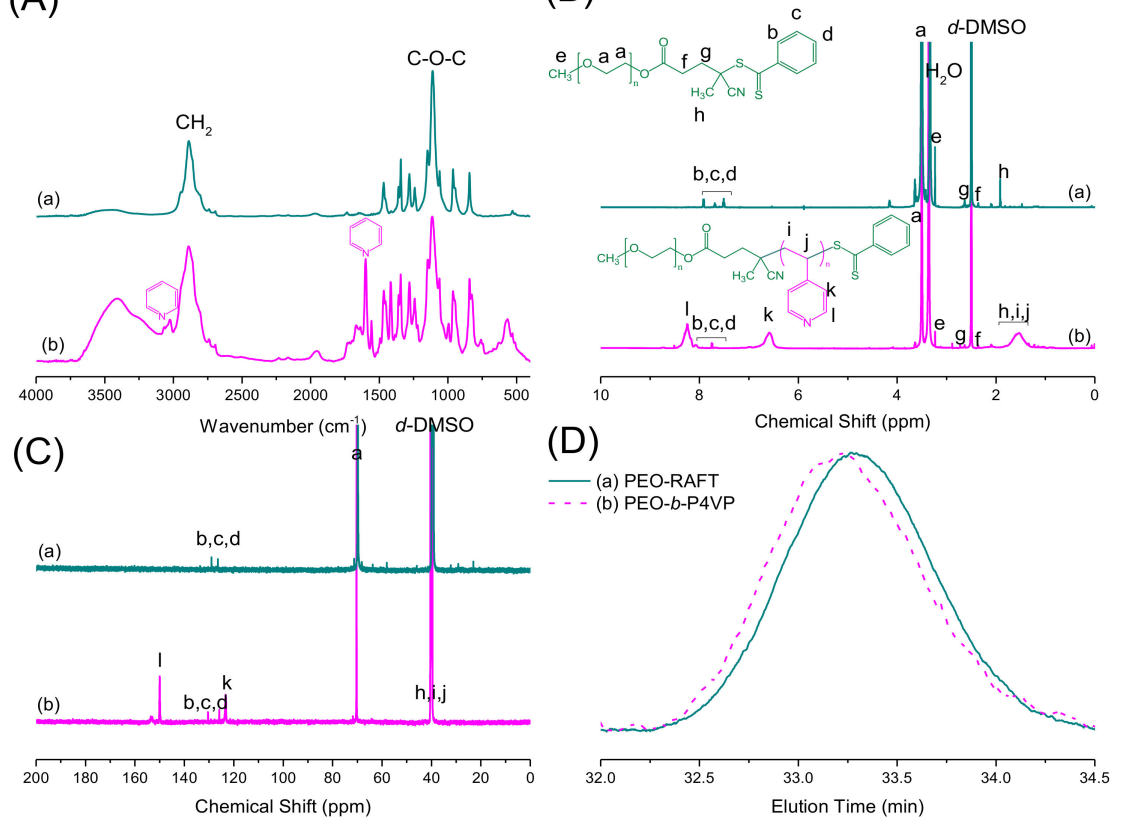

Figure 2. (A) FTIR, (B) ${ }^{1} \mathrm{H},(\mathbf{C}){ }^{13} \mathrm{C}-\mathrm{NMR}$, and (D) GPC analyses of (a) PEO-RAFT and (b) PEO- $b$-P4VP.

After the RAFT polymerization to form PEO-b-P4VP diblock copolymer, signals at 6.59 and 8.25 ppm corresponding to the pyridine units appear, as shown in Figure $2 \mathrm{~B}(\mathrm{~b})$. In addition, 
the ${ }^{13} \mathrm{C}$-NMR spectrum of PEO- $b$-P4VP diblock copolymer is displayed in Figure $2 \mathrm{C}$, where the $\mathrm{CH}_{2}$ unit of the PEO segment is located at $70.0 \mathrm{ppm}$ (Figure $2 \mathrm{C}(\mathrm{a})$ ) and the aromatic rings from the RAFT agent and the P4VP segment are distributed at 122 149 ppm (Figure 2C(b)). The GPC analyses (Figure 2D) of PEO-RAFT macro-initiator featured a mono-modal curve and narrow polydispersity, and after the RAFT polymerization, the signal was shifted to the lower retention time and also displayed a narrow polydispersity, implying the formation of PEO- $b$-P4VP diblock copolymer. We also could determine the number average molecular weight $(\mathrm{Mn})$ and polydispersity of $\mathrm{PEO}_{220}-b-\mathrm{P}_{4} \mathrm{VP}_{82}$ as $18700 \mathrm{~g} /$ mole and 1.11 based on ${ }^{1} \mathrm{H}-\mathrm{NMR}$ and GPC analyses.

\subsection{Self-Assembled Structure of PS-b-PVPh/PEO Diblock Copolymer/Homopolymer Mixtures}

To understand the self-assembly behavior and completive hydrogen bonding interactions of PS- $b$-PVPh/PEO- $b$-P4VP mixtures, we should understand the corresponding behavior of individual PS- $b$-PVPh/PEO and PS- $b$-PVPh/P4VP blends. In our previous studies [11,13], we have investigated the self-assembly structures of PS- $b-\mathrm{PVPh} / \mathrm{P} 4 \mathrm{VP}$ blends, which exhibit the wet-brush behavior featuring fully order-order morphological transition from lamellae, gyroid, cylindrical, and BCC spherical nanostructures. As a result, we firstly investigated the phase behavior of the $\mathrm{PS}_{224}-b-\mathrm{PVPh}_{854} / \mathrm{PEO}_{220}$ blend system. Figure 3 displays the second heating scan of the DSC thermograms (Figure $3 \mathrm{~A}$ ) and $\mathrm{CH}_{2}$ wagging vibration of the PEO segment based on FTIR analyses (Figure 3B) of the PS- $b$-PVPh/PEO blend system. Figure 3A(a) displays the melting temperature of pure PEO at $53{ }^{\circ} \mathrm{C}$ and pure PS- $b$-PVPh displays two $T_{g}$ values at $188^{\circ} \mathrm{C}$ for the PVPh segment and $107^{\circ} \mathrm{C}$ for the PS segment (Figure $1 \mathrm{~A}(\mathrm{f})$ ), suggesting the microphase separation of the PS- $b$-PVPh diblock copolymer. We also observe that the melting temperature $\left(T_{\mathrm{m}}\right)$ and enthalpy of melting of PEO was decreased upon increasing the PS- $b$-PVPh concentrations due to the hydrogen bonding interaction of PVPh/PEO miscible phase for thermodynamic reasons and the morphology effect. The depression of PEO crystalline phase was also investigated by FTIR analyses as displayed in Figure 3B.

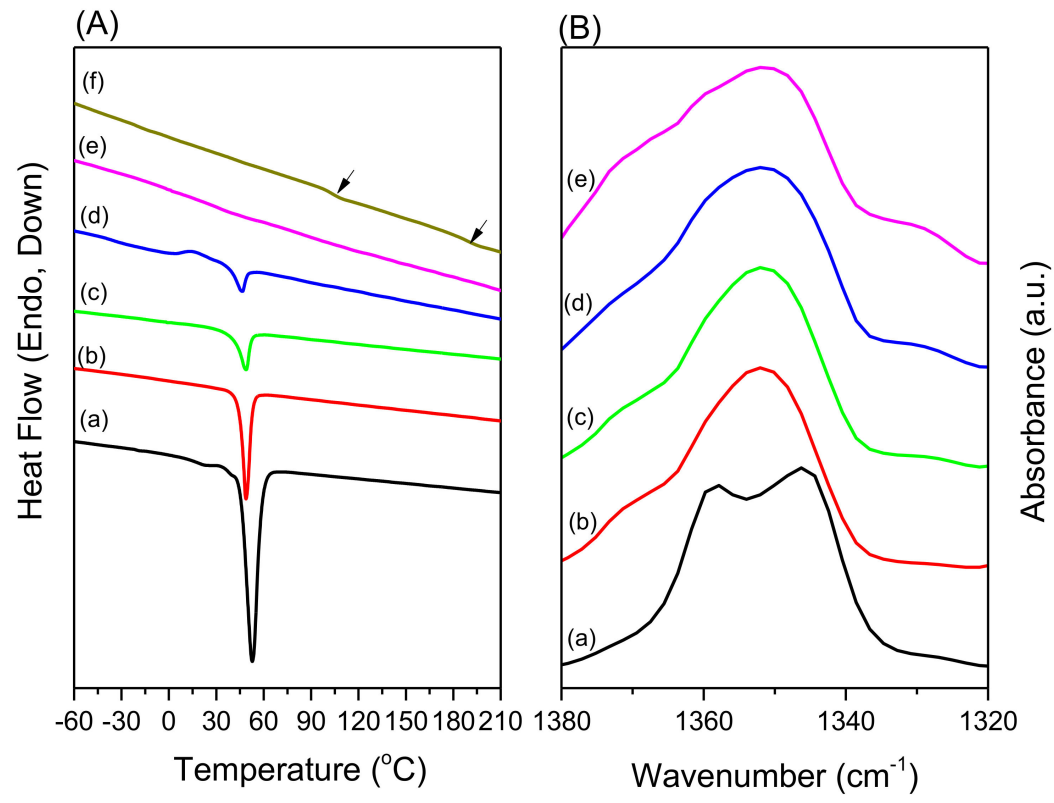

Figure 3. (A) DSC and (B) FTIR analyses of PS- $b$-PVPh/PEO blends with different PEO weight percent (a) 100, (b) 80, (c) 60, (d) 40, (e) 20, and (f) 0 wt.\%.

The absorptions at 1343 and $1360 \mathrm{~cm}^{-1}$ were related to the PEO crystalline phase and these two absorptions disappeared and were replaced by a broad absorption at $1350 \mathrm{~cm}^{-1}$ due to the PEO amorphous phase upon increasing the PS- $b$-PVPh concentrations [41]. As a result, the PEO crystallization is inhibited and retarded upon increasing the amorphous PS- $b$-PVPh diblock copolymer. 
Figure 4 shows SAXS and TEM analyses for the PS- $b$-PVPh/PEO blend system with various PEO compositions at room temperature. Pure PS- $b$-PVPh displayed a lamellar structure long range order as displayed in Figure 4a from the scattering peak ratios of 1:2:3:4:5:6, which could be confirmed by the TEM image (Figure 4e). As the PEO composition was increased to $20 \mathrm{wt} \%$, the lamellar structure still remained, with a scattering ratio of 1:3:5:7, which is also consistent with the TEM image (Figure 4f). Since the even-order peaks (such as 2, 4, and 6) almost disappeared, it implied that the PS volume fraction is close to 0.5 in this case $\left(f_{\mathrm{PS}}{ }^{\mathrm{V}}=0.51\right.$ at this blend composition). Furthermore, the first scattering peak was shifted to a relatively lower value, from $q=0.0885 \mathrm{~nm}^{-1}(d=70.96 \mathrm{~nm})$ to $q=0.0763 \mathrm{~nm}^{-1}$ $(d=82.30 \mathrm{~nm})$, suggesting an increase of the block copolymer spacing. This phenomenon could also be explained by the fact that the PS block segment goes from an asymmetric to a symmetric composition, suggestion that the PEO segment could be dissolved in the PVPh block segment through intermolecular hydrogen bonding interactions and change the volume fraction of the block copolymer. Furthermore, the SAXS peak ratio was observed at $1: \sqrt{ } 3: \sqrt{ } 4: \sqrt{ } 7: \sqrt{ } 9$, representing the hexagonally packed cylindrical nanostructure at $40 \mathrm{wt} \%$ PEO composition as displayed in Figure 4c, and confirmed by the TEM image (Figure $4 \mathrm{~g})$. The first scattering peak was further shifted to a lower $q$ value $\left(q=0.0647 \mathrm{~nm}^{-1}\right.$, $d=97.06 \mathrm{~nm}$ ), also suggesting the increase of block copolymer spacing. However, it displayed wormlike or disordered micelle structures at $60 \mathrm{wt} \%$ PEO composition, where the first scatting peaks was observed and the broad peak ratio of $\sqrt{ } 7$, as displayed in Figure $4 d$, as confirmed by the TEM image (Figure $4 \mathrm{~h}$ ). Furthermore, we also observed a crystalline lamellar structure for peak ratio of 1:2 where the $q$ value of $\left(q_{1}=0.58 \mathrm{~nm}^{-1}, d=10.82 \mathrm{~nm}, q_{2}=1.16 \mathrm{~nm}^{-1}, d=5.41 \mathrm{~nm}\right)$ and thus the self-assembly structure from miscible PVPh/PEO phase also competed with the crystallization behavior of the PEO domain in this case. Overall, we could conclude that the PS- $b$-PVPh/PEO blends also display the wet-brush behavior where $K_{\mathrm{A}}=280$ of $\mathrm{PVPh} / \mathrm{PEO}$ is stronger than the $K_{\mathrm{B}}=66.8$ of PVPh, which is consistent with our previous proposed scheme. However, it did not display a full order-order morphological transition, probably because of the relatively weaker intermolecular hydrogen bonding strength as compared with strong PVPh/P4VP blend system $\left(K_{\mathrm{A}}=1200\right)$ [11-13,42-45].
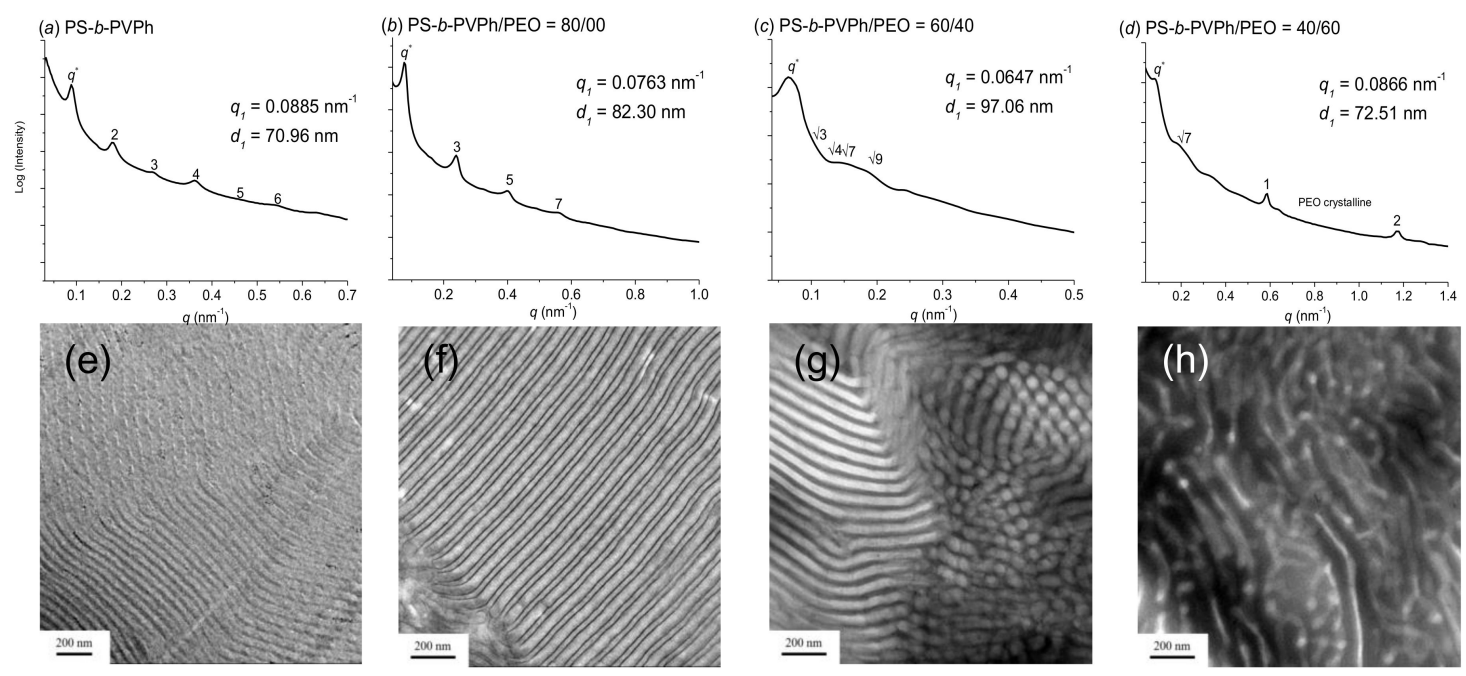

Figure 4. SAXS and TEM images of PS- $b$-PVPh/PEO blends of $(\mathbf{a}, \mathbf{e}) 100 / 0,(\mathbf{b}, \mathbf{f}) 80 / 20,(\mathbf{c}, \mathbf{g}) 60 / 40$, and (d,h) 40/60.

\subsection{Self-Assembled Structure of PS-b-PVPh/PEO-b-P4VP Diblock Copolymer Mixtures}

In this study, we prepared two different diblock copolymers by using anionic living $\left(\mathrm{PS}_{158}-b-\mathrm{PVPh}_{460}\right)$ and RAFT (PEO-b-P4VP) polymerizations with hydrogen bonded donor or acceptor groups as displayed in Scheme 1. The molecular weights, solubility parameters, molar volumes, equilibrium constants, and interaction parameters of each block segment are summarized in Table 1 and Scheme 2. 
Table 1. Molecular volume, molecular weight, solubility parameter, and equilibrium constants of various polymer segments used in this work.

\begin{tabular}{|c|c|c|c|c|c|c|}
\hline \multirow[t]{2}{*}{ Polymer } & \multirow{2}{*}{$\begin{array}{c}\text { Molar Volume } \\
(\mathrm{mL} / \mathrm{mol})\end{array}$} & \multirow{2}{*}{$\begin{array}{c}\text { Molecular Weight } \\
(\mathrm{g} / \mathrm{mol})\end{array}$} & \multirow{2}{*}{$\begin{array}{c}\text { Solubility Parameter } \\
\left((\mathrm{cal} / \mathrm{mL})^{1 / 2}\right)\end{array}$} & \multicolumn{3}{|c|}{ Equilibrium Constants } \\
\hline & & & & $K_{2}$ & $K_{\mathrm{B}}$ & $K_{\mathrm{A}}$ \\
\hline PVPh & 82.3 & 120.1 & 11.0 & 21.0 & 66.8 & \\
\hline PS & 93.9 & 104.1 & 9.5 & - & - & - \\
\hline PEO & 38.1 & 44.1 & 9.40 & - & - & 280 \\
\hline P4VP & 84.9 & 105.1 & 10.8 & - & - & 1200 \\
\hline
\end{tabular}

(a)

(b)
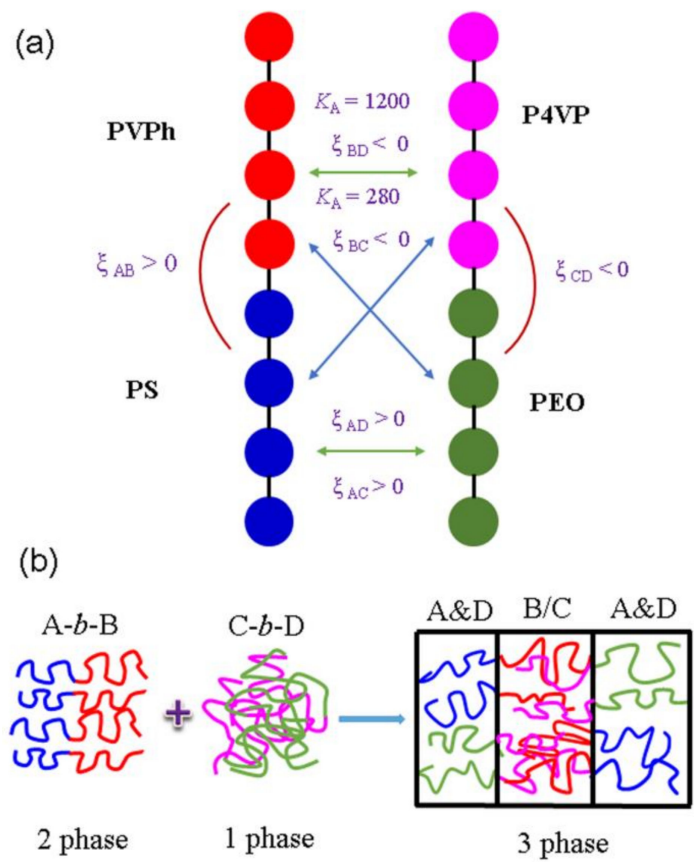

Scheme 2. (a) Inter-association equilibrium constant and interaction parameters of PS- $b$-PVPh/PEO- $b$-P4VP mixtures and (b) cartoon representation of self-assembly nanostructures for $\mathrm{A}-b-\mathrm{B} / \mathrm{C}-b-\mathrm{D}$ mixtures in this study.

Molecular volume, molecular weight, solubility parameter, self-association dimer $\left(K_{2}\right)$ and multimer $\left(K_{\mathrm{B}}\right)$, and inter-association $\left(K_{\mathrm{A}}\right)$ equilibrium constants were determined based on reference [44].

As shown in Scheme 2, six different interaction parameters are expected in PS- $b$-PVPh/PEO- $b$-P4VP mixtures. Pure PS- $b$-PVPh block copolymers display the typical upper critical ordering transition (UCOT) phase behavior, implying that the microphase separation was occurred at lower temperature (Figure 4(a)) and become disordered structures at a higher temperature (order-to-disorder transition (ODT)) because of reduction the interaction parameter $(\chi)$ with the increase of temperature, which was observed for most diblock copolymer systems. However, Chen et al. have proposed that PEO-b-P4VP diblock copolymers possess a lower critical ordering transition (LCOT), which is similar to the lower critical solution temperature (LCST) in polymer blend systems [46]. This disorder-to-order transition (DOT) was rarely observed in diblock copolymer systems, implying that the PEO- $b$-P4VP diblock copolymer displays a disordered structure at lower temperature. As a result, three interaction parameters would be negative including $\mathrm{PVPh} / \mathrm{P} 4 \mathrm{VP}, \mathrm{PVPh} / \mathrm{PEO}$, and PEO/P4VP miscible or disorder binary blends and the other three interaction parameters would be positive indicating that PS/PEO, PS/PVPh, and PS/P4VP are immiscible binary blends However, the $K_{\mathrm{A}}$ value of the PVPh/P4VP (1200) [42-45] is much greater than that of PVPh/PEO $\left(K_{\mathrm{A}}=280\right)$ [44] indicating that the PVPh would tend to interact more with the P4VP segment than the PEO segment. Mediating this competitive hydrogen bonding 
strength with different blend compositions, the PEO block might possess microphase separation from disordered PEO-b-P4VP diblock copolymer during blending with PS- $b$-PVPh and then induce three phases (PS, PVPh/P4VP, and PEO phases) or even hierarchical self-assembled structures as displayed in Scheme $2 b$. DSC analysis is widely used for understanding the miscibility behavior of polymer blends. Figure 5A shows the heating thermograms of PS- $b$-PVPh/PEO- $b$-P4VP blends with various compositions based on DSC analyses from $-60{ }^{\circ} \mathrm{C}$ to $210^{\circ} \mathrm{C}$. Similarly, pure PS-b-PVPh also displays two $T_{\mathrm{g}}$ values at $188^{\circ} \mathrm{C}$ for PVPh segment and $105{ }^{\circ} \mathrm{C}$ for PS segment (Figure 5(a)) and pure PEO-b-P4VP diblock copolymer displays a $T_{\mathrm{m}}$ value at $61^{\circ} \mathrm{C}$ for the PEO segment and a $T_{\mathrm{g}}$ value at $140{ }^{\circ} \mathrm{C}$ for the P4VP segment (Figure 5(f)). It was difficult to observe the $T_{\mathrm{g}}$ value for the PEO segment in this study because of the high crystallinity behavior of this diblock copolymer. Compared with the PS- $b-\mathrm{PVPh} / \mathrm{PEO}$ blend system, the melting temperature of the PEO block segment in PEO- $b$-P4VP almost remained constant with the increase of PS- $b$-PVPh concentration $(<70 \mathrm{wt} \%)$, indicating that the phenolic $\mathrm{OH}$ unit of PVPh did not interact with the ether units of PEO in PS- $b$-PVPh/PEO- $b$-P4VP mixtures at these compositions. Due to the high crystallinity behavior of PEO block segment, we expanded the DSC thermograms from $80^{\circ} \mathrm{C}$ to $210^{\circ} \mathrm{C}$ to avoid the melting behavior of the PEO segment.

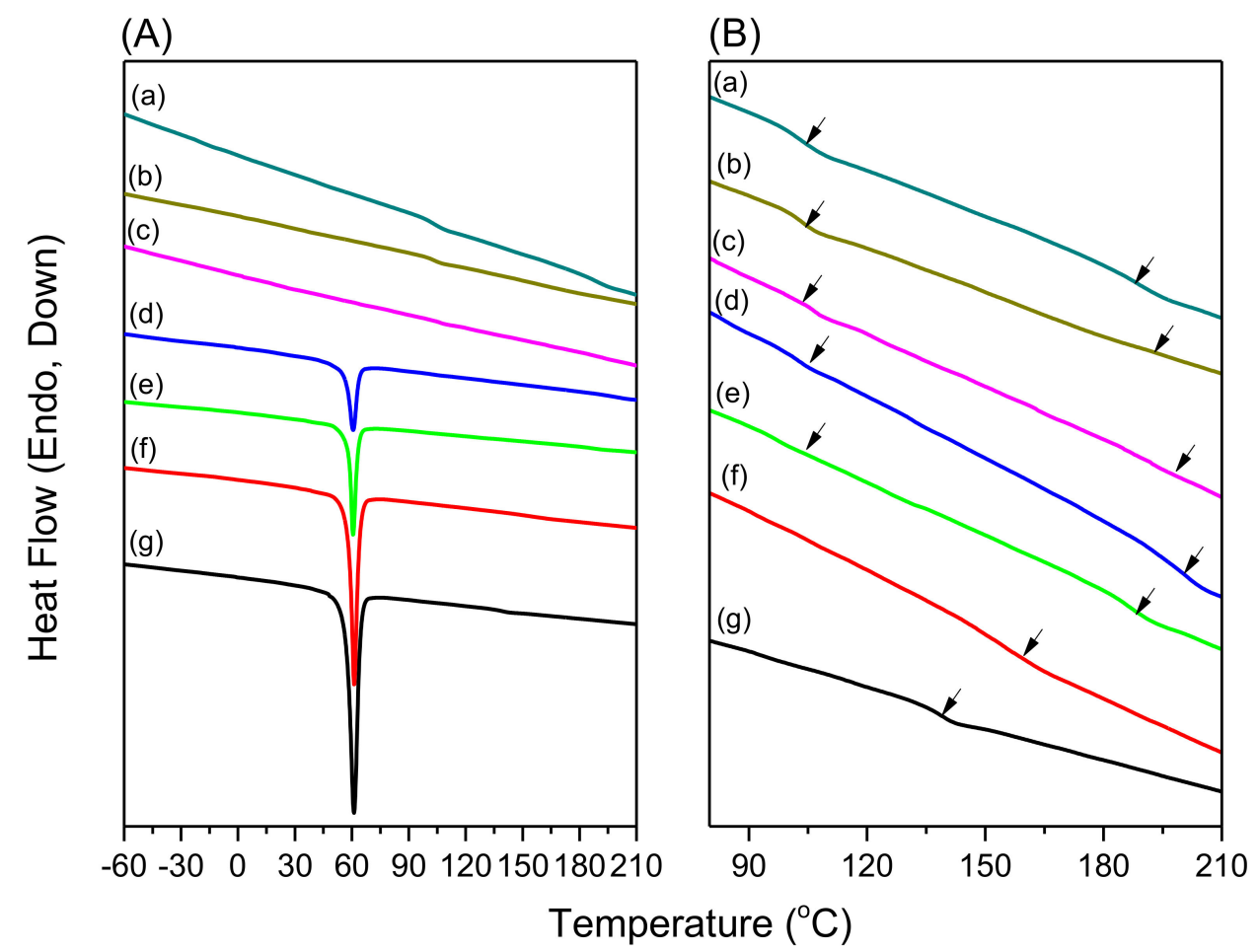

Figure 5. DSC analyses of PS- $b$-PVPh/PEO- $b$-P4VP mixtures of range from (A) $-60 \sim 210{ }^{\circ} \mathrm{C}$, and (B) $80 \sim 210{ }^{\circ} \mathrm{C}$ for (a) 100/0, (b) 90/10, (c) 70/30, (d) 50/50, (e) 30/70, (f) 10/90, and (g) 0/100.

As shown in Figure 5B. Clearly, we found two $T_{\mathrm{g}}$ values for all diblock copolymer mixture compositions, suggesting that microphase separation occurred for these diblock copolymer mixtures. The lower $T_{\mathrm{g}}$ values $\left(105-106^{\circ} \mathrm{C}\right)$ corresponded to the PS segment, while the higher $T_{\mathrm{g}}$ values $(161-202$ ${ }^{\circ} \mathrm{C}$ ) are due to the miscible $\mathrm{PVPh} / \mathrm{P} 4 \mathrm{VP}$ domain through strong hydrogen bonding interactions, appear higher than both individual homopolymers at lower PEO- $b$-P4VP blend compositions. Furthermore, these $T_{\mathrm{g}}$ values were also higher than the binary homopolymer blend of $\mathrm{PVPh} / \mathrm{P} 4 \mathrm{VP}=1 / 1$ from DMF solution $\left(T_{\mathrm{g}}=190^{\circ} \mathrm{C}\right)$ presumably due to the nanoconfinement effect from microphase separation of the diblock copolymer mixtures in this study. As a result, we could observe the higher $T_{\mathrm{g}}$ behavior at $161-202{ }^{\circ} \mathrm{C}$ from the miscible PVPh/P4VP domain, the lower $T_{\mathrm{g}}$ values at $105-106{ }^{\circ} \mathrm{C}$ from PS 
segment, and a melting temperature at ca. $60^{\circ} \mathrm{C}$ for the PEO segment, indicating that at least three phases existed for the PS, PVPh/P4VP, and PEO domains in this PS- $b$-PVPh/PEO- $b$-P4VP blend.

Figure 6 summarizes $T_{\mathrm{g}}$ behavior of the PS domain and PVPh/P4VP domain and $T_{\mathrm{m}}$ behavior of the PEO domain for PS- $b$-PVPh/PEO-b-P4VP blends. Clearly, the $T_{\mathrm{g}}$ value of PS domain and $T_{\mathrm{m}}$ value of PEO domain almost did not change with the various blend compositions; however, the $T_{\mathrm{g}}$ value of $\mathrm{PVPh} / \mathrm{P} 4 \mathrm{VP}$ domain displayed positive deviations based on the linear rule and these values could be predicted by the Kwei equation for strong hydrogen bonded blend systems [47]:

$$
T_{g}=\frac{W_{1} T_{g 1}+k W_{2} T_{g 2}}{W_{1}+k W_{2}}+q W_{1} W_{2}
$$

where $T_{\text {gi }}$ represents the glass transition temperature of each segment, $W_{i}$ represents each weight fraction of block segment; $k$ and $q$ are due to the fitting constant. We can determine the $k$ and $q$ values of 1 and 150, which represents the strong hydrogen bonding interaction in this work.

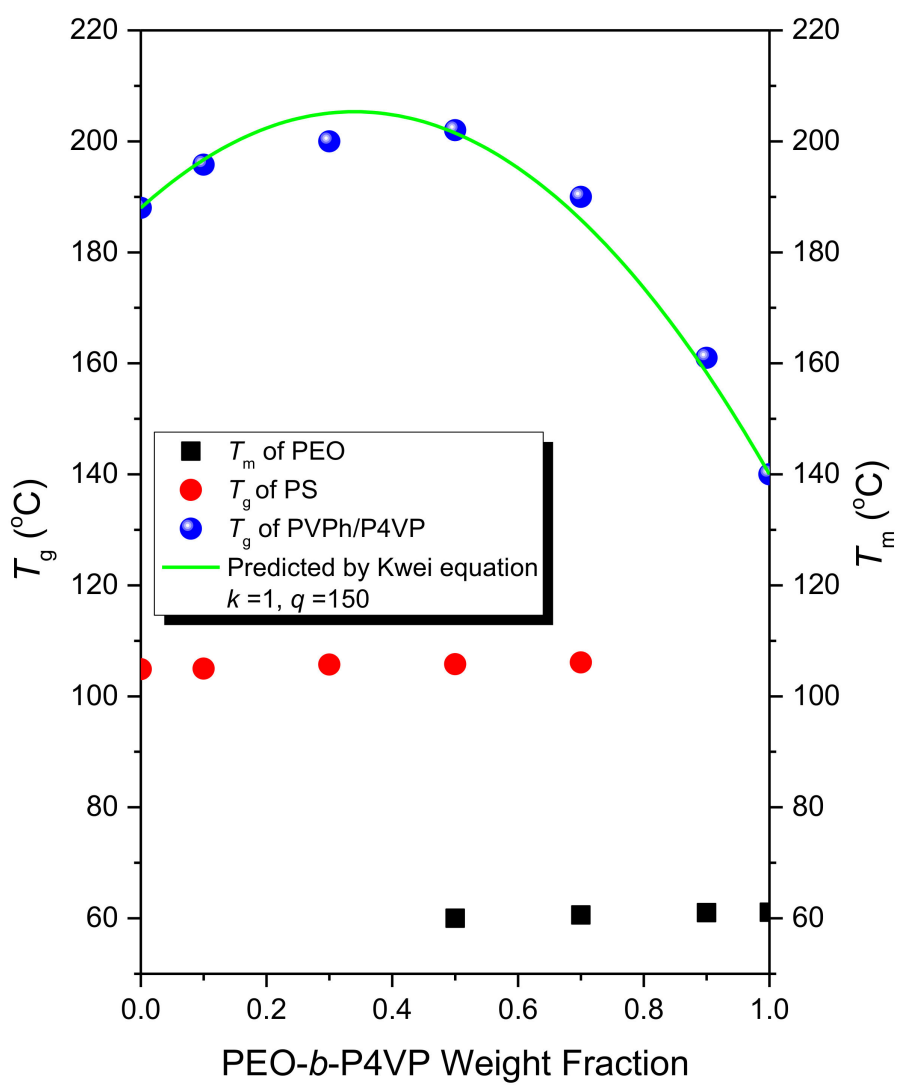

Figure 6. $T_{\mathrm{g}}$ behavior of PS and PVPh/P4VP domain (also predicted by Kwei equation), and $T_{\mathrm{m}}$ behavior of PEO segment of PS- $b$-PVPh/PEO- $b$-P4VP mixtures.

We used FTIR spectroscopy to investigate the intermolecular hydrogen bonding interaction and crystallization behavior of the PS- $b$-PVPh/PEO- $b$-P4VP diblock copolymer mixture as displayed in Figure 7. Figure 7A displays the FTIR spectra of pure PEO- $b$-P4VP for various PS- $b$-PVPh weight compositions as inferred from the 1380 to $1320 \mathrm{~cm}^{-1}$ region due to the $\mathrm{CH}_{2}$ wagging of the $\mathrm{PEO}$ segment. 


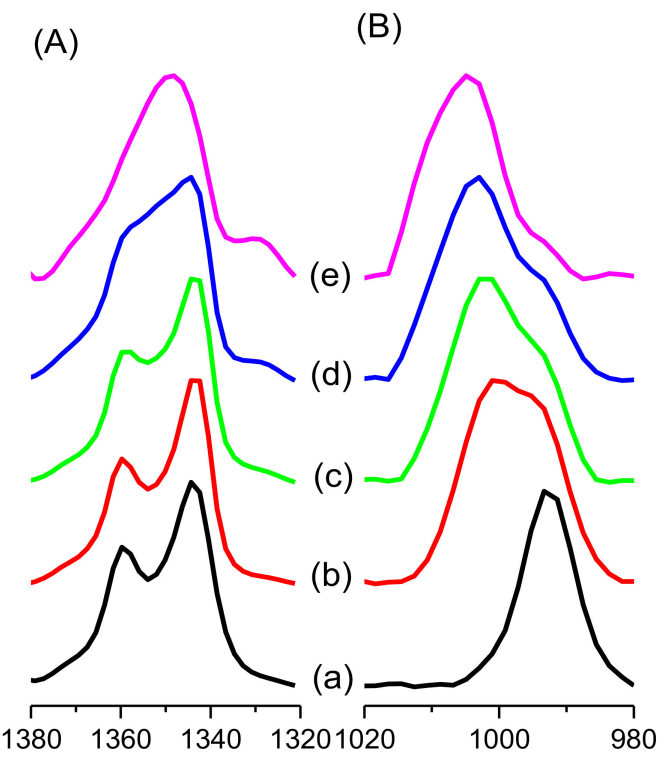

(C)

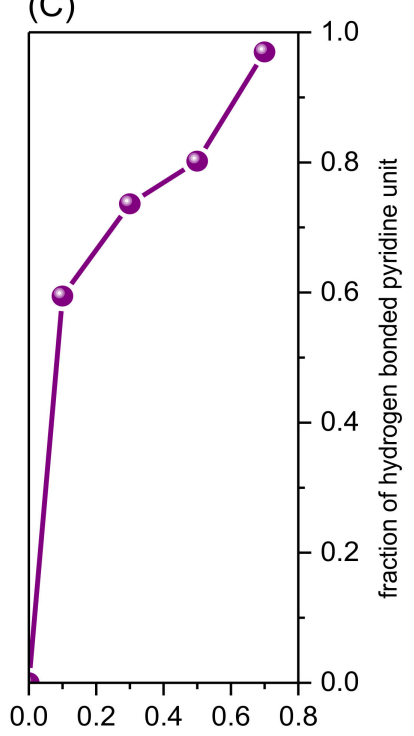

Figure 7. FTIR analyses of PS- $b$-PVPh/PEO-b-P4VP mixture of (A) $\mathrm{CH}_{2}$ wagging, (B) pyridine vibration of (a) 0/100, (b) 10/90, (c) 30/70, (d) 50/50, (e) 70/30, and (C) fraction of hydrogen bonded pyridine unit with different PS- $b$-PVPh weight fractions.

Similar to Figure 3B, the absorptions at 1343 and $1360 \mathrm{~cm}^{-1}$ represent the PEO crystalline phase and these two absorptions disappear and were replaced by a broad absorption at $1350 \mathrm{~cm}^{-1}$ due to the PEO amorphous phase at $70 \mathrm{wt} \%$ of PS- $b$-PVPh concentrations, which is consistent with the DSC analyses where the melting peak disappeared for this blend composition. The result is also consistent with the WAXD analyses as displayed in Figure 8. Two strong (120) $\mathrm{PEO}$ and (032) PEO diffraction peaks for pure PEO-b-P4VP diblock copolymer were observed in Figure 8(a) and they disappeared at $70 \mathrm{wt} \%$ of PS- $b$-PVPh concentration. Furthermore, Figure 7B exhibits the absorption at $993 \mathrm{~cm}^{-1}$ for the free pyridine units of the P4VP segment and a new absorption at $1005 \mathrm{~cm}^{-1}$ was observed for the hydrogen bonded pyridine unit in the PVPh/P4VP domain. We determined the fraction hydrogen bonded pyridine units based on the digital subtraction at $1013 \mathrm{~cm}^{-1}$ due to the PVPh unit by considering the molar faction of PVPh block and we also observed that the value of fraction of hydrogen bonded pyridine unit was increased with the increase of PS- $b-\mathrm{PVPh}$ concentration as displayed in Figure 7C.

Figure 9 displays SAXS analyses for PS- $b$-PVPh/PEO- $b$-P4VP blends with different compositions at room temperature. Pure PS- $b$-PVPh as displayed in Figure 9A-(a) also shows the long range order of a lamellar structure based on the scattering peak ratio of 1:2:3:4:6 and the corresponding $d$-spacing lamellar structure is $50.2 \mathrm{~nm}$ from the first peak position at $q^{*}=0.125 \mathrm{~nm}^{-1}$. The TEM image as displayed in Figure 10a also reveals that the pure PS- $b-\mathrm{PVPh}$ shows a lamellar structure, which is consistent with the SAXS pattern. In addition, pure PEO- $b$-P4VP diblock copolymer displays a disordered structure at lower temperature as shown in Figure $9(\mathrm{~g})$ without any peak, as also confirmed by the TEM image (Figure 10g). 


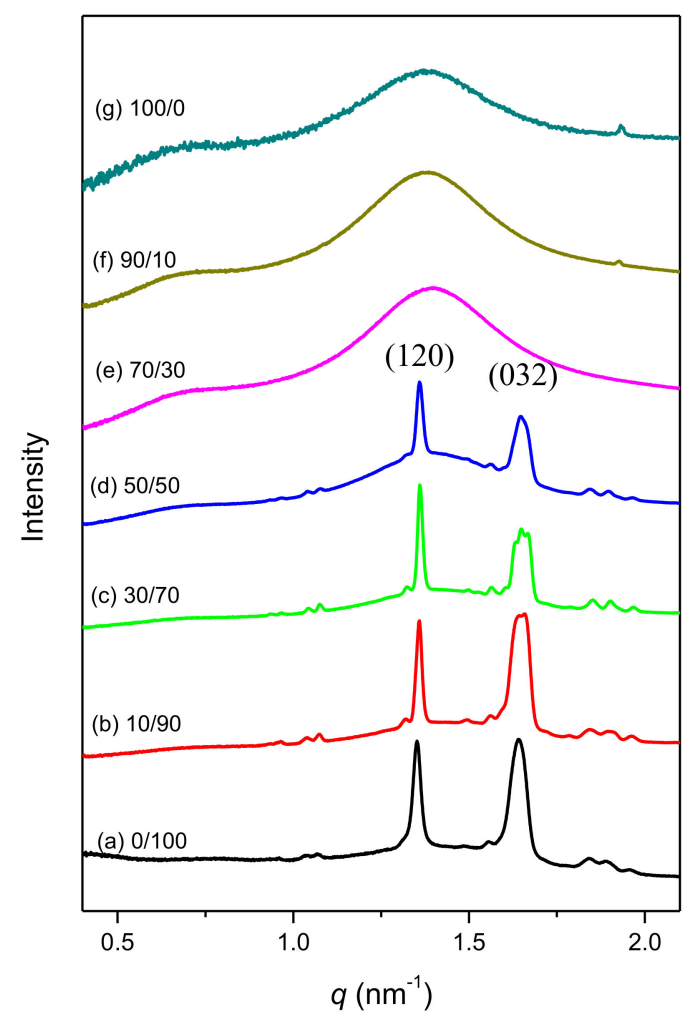

Figure 8. WAXD analyses of PS- $b$-PVPh/PEO- $b$-P4VP mixture at room temperature of (a) 0/100, (b) 10/90, (c) 30/70, (d) 50/50, (e) 70/30, (f) 90/10, and (g) 100/0.

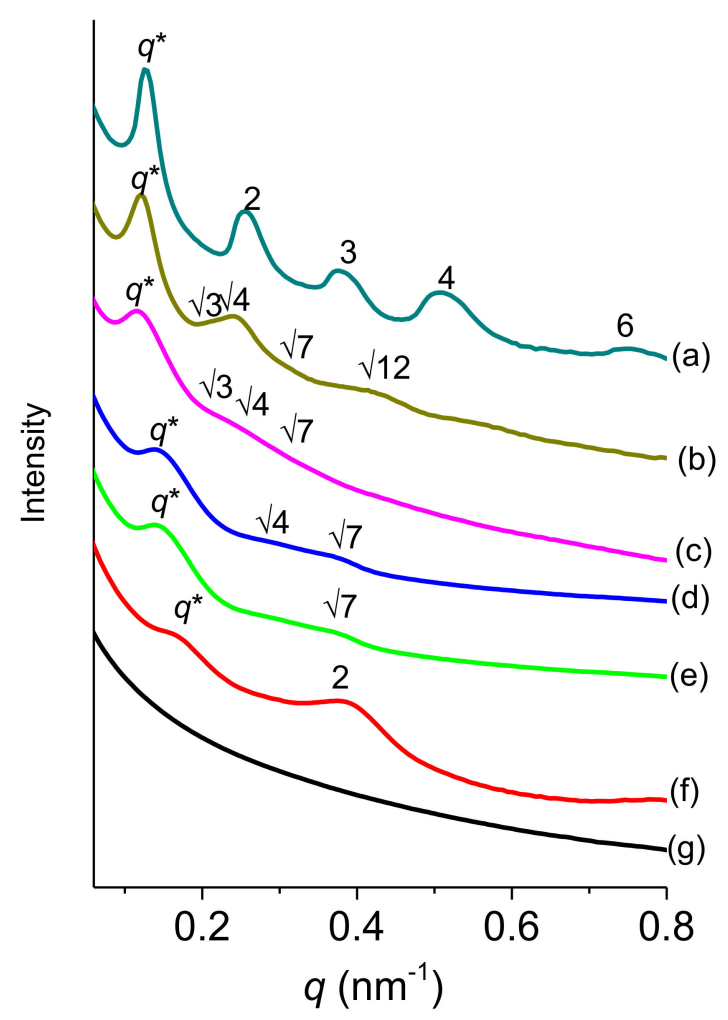

Figure 9. SAXS analyses of PS- $b$-PVPh/PEO- $b$-P4VP mixture at room temperature of (a) 0/100, (b) 10/90, (c) 30/70, (d) 50/50, (e) 70/30, (f) 90/10, and (g) 100/0. 
As the PEO- $b$-P4VP concentration increases to $10 \mathrm{wt} \%$, the SAXS pattern displays a highly ordered cylindrical structure with a scattering ratio of $1: \sqrt{ } 3: \sqrt{ } 4: \sqrt{ } 7: \sqrt{ } 12$, as also consistent with the TEM analysis (Figure 10b) and the first scattering peak was shifted slightly to a $q$ value at $0.121 \mathrm{~nm}^{-1}$ $(d=51.9 \mathrm{~nm})$. Further increasing the PEO- $b$-P4VP concentrations to 30 or $50 \mathrm{wt} \%$ also results in cylindrical structures based on the peak ratios of $1: \sqrt{ } 3: \sqrt{ } 4: \sqrt{ } 7$ as shown in Figures $9 \mathrm{c}, \mathrm{d}$ and were also consistent with the TEM images in Figures 10c,d. The first scattering peaks appeared at $q$ values of $0.117 \mathrm{~nm}^{-1}(d=53.67 \mathrm{~nm})$ at $30 \mathrm{wt} \%$ PEO- $b$-P4VP and $0.137 \mathrm{~nm}^{-1}(d=45.83 \mathrm{~nm})$ at $50 \mathrm{wt} \%$ PEO- $b$-P4VP. Clearly, the addition of the PEO- $b$-P4VP diblock copolymer could induce the order-order transition from lamellae to cylindrical structures featuring the wet-brush behavior. Upon further increasing the $\mathrm{PEO}-b$-P4VP concentration to $70 \mathrm{wt} \%$, a disordered wormlike structure with a peak ratio of $1: \sqrt{ } 7$ is displayed as shown in Figure 9(e), consistent by the TEM image (Figure 10e). As the PEO-b-P4VP concentration increases to $90 \mathrm{wt} \%$, it displays a lamellar structure with a peak ratio of 1:2 as displayed in Figure 9(f), also confirmed by the TEM image (Figure 10f). The lamellar structure may come from the crystallization behavior from the PEO block segment as displayed in the DSC, FTIR, and WAXD analyses. Since the driving force of PEO crystallization behavior may be favored more than the microphase separation of block segments thus the crystalline lamellar structure or short range order micellar structure are observed at relative higher PEO-b-P4VP concentrations. Based on these results, although the PS- $b$-PVPh/P4VP system could exhibit a fully order-order transition from lamellae to gyroid to cylinder and finally to BCC spheres, the PS- $b$-PVPh/PEO- $b$-P4VP mixtures only display the order-order transition from lamellae to hexagonally packed cylinders. The relatively weaker hydrogen bonding strength of the PEO segment compared with the P4VP segment and the crystallization behavior of the PEO segment all inhibit the fully order-order transition for PS- $b-\mathrm{PVPh} / \mathrm{PEO}-b-\mathrm{P} 4 \mathrm{VP}$ mixtures in this study.
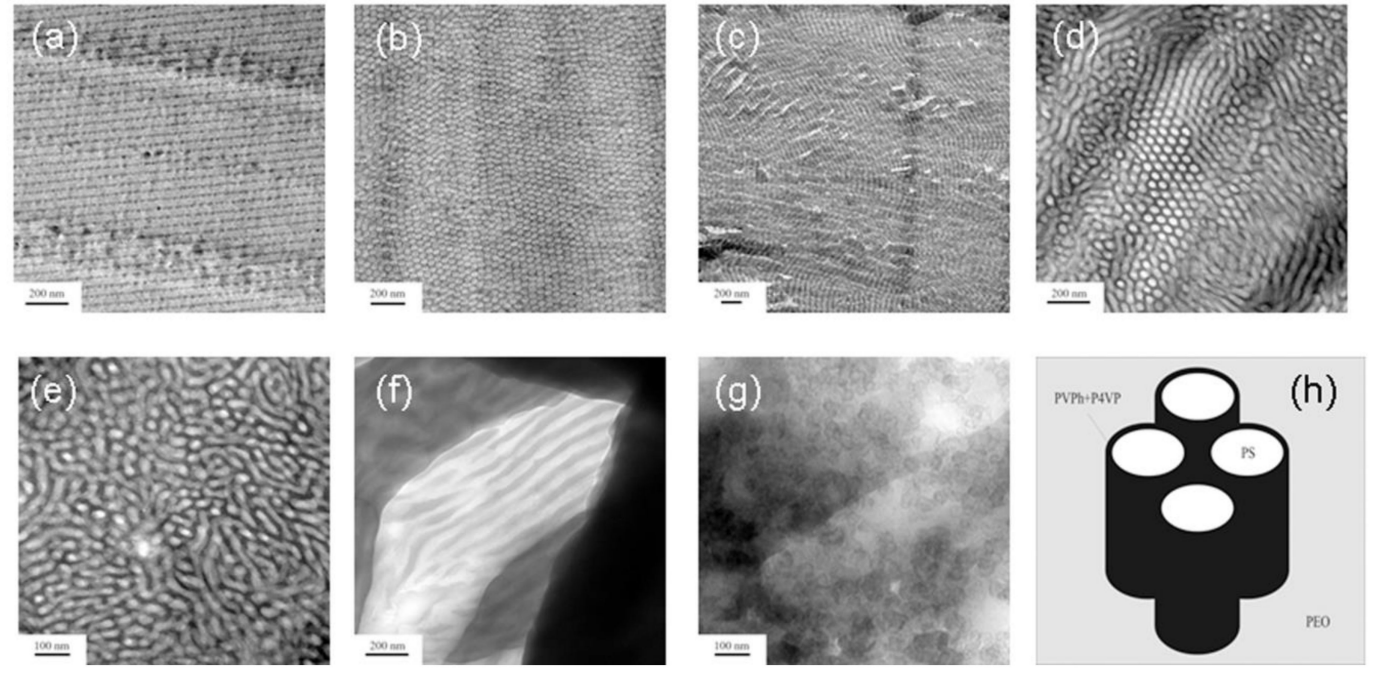

Figure 10. TEM images staining with $\mathrm{I}_{2}$ of PS- $b$-PVPh/PEO- $b$-P4VP mixture at room temperature of (a) 100/0, (b) 90/10, (c) 70/30, (d) 50/50, (e) 30/70, (f) 10/90, (g) 0/100, (h) cartoon representation of core-shell cylinder structure of PS, PVPh/P4VP, and PEO domains.

However, the advantage of PS- $b$-PVPh/PEO- $b$-P4VP mixture system is that it could exhibit three-phase behavior compared with PS- $b$-PVPh/PEO or PS- $b$-PVPh/P4VP diblock copolymer/ homopolymer blends. Here, we used $\mathrm{RuO}_{4}$ for further staining the PS- $b$-PVPh/PEO- $b$-P4VP mixtures. TEM images determined after staining with $\mathrm{I}_{2}$ could reveal the P4VP domains and further staining with $\mathrm{RuO}_{4}$ could provide the PEO domains in this diblock copolymer mixture. Figure 11d,f display TEM images for PS- $b$-PVPh/PEO- $b$-P4VP $=90 / 10,70 / 30$, and 50/50 determined after staining with both $\mathrm{I}_{2}$ and $\mathrm{RuO}_{4}$. Clearly, the PVPh/P4VP domain appears dark; the PS domain is white, and the PEO domain 
appears gray as displayed in Figure 10h. Since the $K_{\mathrm{A}}$ for PVPh/P4VP complex is much larger than that of PVPh/PEO, the PEO segment may be excluded from the disordered PEO- $b$-P4VP block copolymer and thus induce microphase separation. In addition, due to the intrinsic immiscibility behavior of the PEO and PS segments, the three phases of PS- $b$-PVPh/PEO- $b$-P4VP blends as expected show PS, PVPh/P4VP, and PEO domains as seen in Figure 10h.
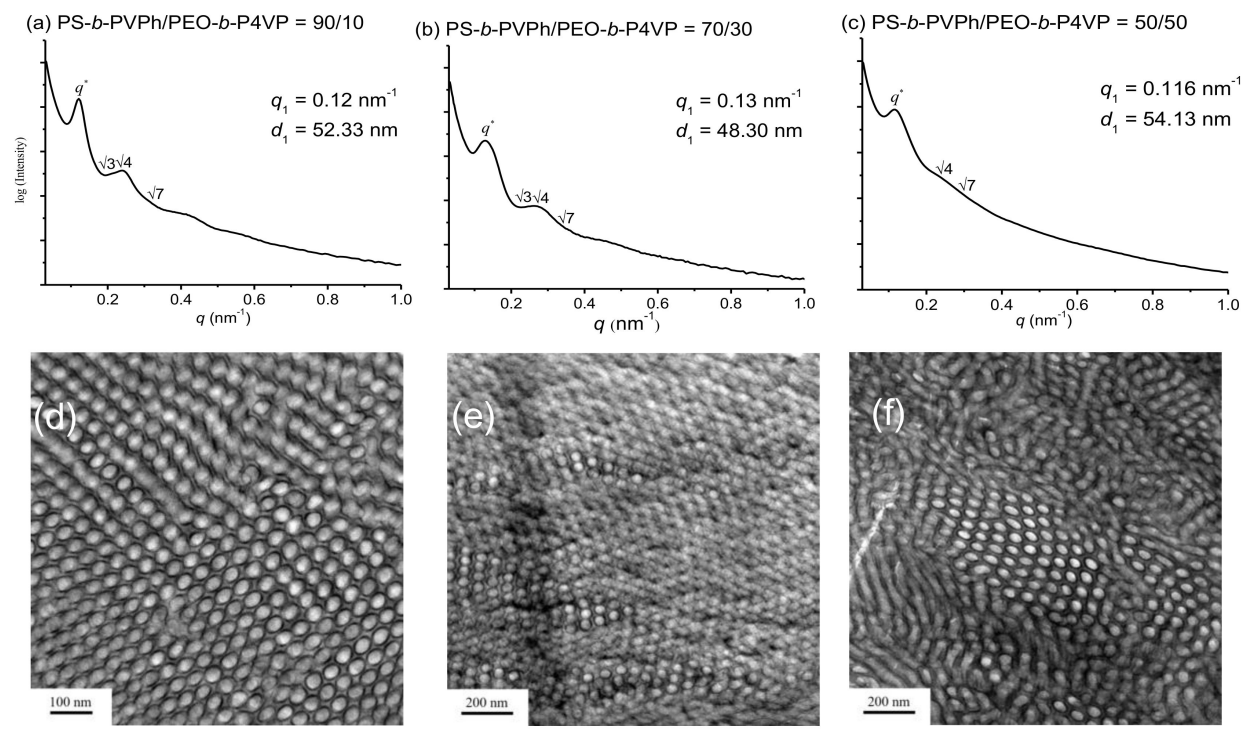

Figure 11. SAXS analyses and TEM images staining with $\mathrm{I} 2$ and $\mathrm{RuO}_{4}$ of PS- $b$-PVPh/PEO- $b$-P4VP mixture mixture at room temperature of $(\mathbf{a}, \mathbf{d}) 90 / 10,(\mathbf{b}, \mathbf{e}) 70 / 30$, and $(\mathbf{c}, \mathbf{f}) 50 / 50$.

\section{Conclusions}

We have successfully prepared PS- $b$-PVPh and PEO- $b$-P4VP by sequential anionic living and RAFT polymerizations according to FTIR, NMR and GPC analyses, respectively. Since the $K_{\mathrm{A}}$ value and hydrogen bonding strength of the $\mathrm{PVPh} / \mathrm{P} 4 \mathrm{VP}$ domain is much greater than those of the $\mathrm{PVPh} / \mathrm{PEO}$ domain thus the PEO block segment was excluded from the disordered PEO- $b$-P4VP and then undergoes microphase separation. In addition, due to the intrinsic immiscible behavior of the $\mathrm{PEO}$ and PS block segments, the PS- $b$-PVPh/PEO- $b$-P4VP blends possesses a hierarchical self-assembled behavior as core-shell cylindrical structures by taking advantage of competitive hydrogen bonding and $\Delta K$ effect in this study.

\section{Experimental Section}

\subsection{Materials}

4-Vinylpyridine (4VP, 95\%, Acros, Ward Hill, MA, USA) was distilled from $\mathrm{CaH}_{2}$. DMF was distilled from $\mathrm{CaH}_{2}$ after the heating under reflux for $4 \mathrm{~h}$ under $\mathrm{N}_{2}$. Poly(ethylene glycol) 4-cyano-4-(phenylcarbonothioylthio) pentanoate (PEO-SC(S)Ph) $\left(M_{\mathrm{n}}=10000 \mathrm{~g} / \mathrm{mol}\right)$ was purchased from Aldrich (St. Louis, MO, USA). The synthesis of PS- $b$-PVPh diblock copolymer was performed according to our previous works $[11,13,40]$

\subsection{The Synthesis of PEO-b-P4VP Diblock Copolymer by Using RAFT Polymerization}

AIBN (23 mg) and PEO-SC(S)Ph (0.3042 g) were mixed in DMF (8.6 mL). After three cycles of freeze-vacuum-thaw degassing process, then 4VP monomer $(0.4 \mathrm{~mL} 3.7 \mathrm{mmol})$ was added and the solution was immersed in an oil bath at $70{ }^{\circ} \mathrm{C}$ under a $\mathrm{N}_{2}$ atmosphere for $24 \mathrm{~h}$. After cooling to $25^{\circ} \mathrm{C}$, the reaction was stopped and the mixture dropped into diethyl ether. The precipitate was filtered and dried under vacuum overnight (yield: $66 \mathrm{wt} \%$ ). 


\subsection{PS-b-PVPh/PEO-b-P4VP Blends}

Different PS- $b$-PVPh/PEO- $b$-P4VP blend compositions are prepared by using solution casting, which are firstly dissolved in DMF since these four block segments are all soluble in DMF ( $5 \mathrm{wt} \%$ ). The block copolymer mixtures were stirred for $48 \mathrm{~h}$ at $25^{\circ} \mathrm{C}$ and were evaporated at $90{ }^{\circ} \mathrm{C}$ slowly for $48 \mathrm{~h}$ and dried under the vacuum at $120^{\circ} \mathrm{C}$ for $96 \mathrm{~h}$ to remove the residual DMF solvent.

\subsection{Characterizations}

FTIR spectroscopy of these blend samples were measured through the conventional $\mathrm{KBr}$ disk method and a Tensor 27 spectrophotometer (Bruker, Billerica, MA, USA) with 32 scans and a spectral resolution of $4 \mathrm{~cm}^{-1}$ were used. Molecular weights and their corresponding polydispersities of block copolymers were determined through gel permeation chromatography (GPC) using a Waters 510 HPLC system (GPC, Waters, Taipei, Taiwan) and DMF was used as the eluent. The calibration curve was constructed by PS standard with the combinations of refractive index, UV, viscosity, and light scattering detectors. NMR spectra were recorded on an AM 500 spectrometer (Bruker, Billerica, MA, USA) using $\mathrm{CDCl}_{3}$ or $d$-DMSO as $d$-solvents. Thermal properties are measured using a TA Q-200 instrument (TA Instrument, New Castle, DE, USA) from room temperature to $260^{\circ} \mathrm{C}$ at $20^{\circ} \mathrm{C} / \mathrm{min}$ heating rate. SAXS analyses of these diblock copolymer mixtures were recorded using the BL23A1 $(\lambda=1.1273 \AA$ ) beamline (National Synchrotron Radiation Research Center, NSRRC), Taiwan. In general, blend specimens are sealed between Kapton films and determined at room temperature. TEM analyses of blend samples were performed using a JEOL 2100 microscope (Tokyo, Japan), which was operated at $200 \mathrm{kV}$. The diblock copolymer mixture films were cut into ultrathin sections by an ultra-cut microtome (Leica, Taipei, Taiwan) equipped with a diamond knife. The TEM images were determined after staining with $\mathrm{I}_{2}$ to exhibit the P4VP segments and further stained with $\mathrm{RuO}_{4}$ to find the PVPh and PEO domains. Thus, the PVPh/P4VP domains appear dark, the PEO domains appear gray, while the PS domains appear white in this diblock copolymer mixture.

Author Contributions: T-C.T. contributed to the synthesis of block copolymers; and S-W.K. coordinated the study, interpreted the results, and wrote the paper.

Funding: This study was supported financially by the Ministry of Science and Technology, Taiwan, under contracts MOST 106-2221-E-110-067-MY3 and 105-2221-E-110-092-MY3.

Conflicts of Interest: The authors declare no conflict of interest.

\section{References}

1. Jenekhe, S.A.; Chen, X.L. Self-assembly of ordered microporous materials from rod-coil block copolymers. Science 1999, 283, 372-375. [CrossRef] [PubMed]

2. Guiod, A.R.; Vandermeulen, W.M.; Klok, V.H. Advanced drug delivery devices via self-assembly of amphiphilic block copolymers. Adv. Drug Deliv. Rev. 2012, 64, 270-279.

3. Lin, E.L.; Hsu, W.L.; Chiang, Y.W. Trapping structural coloration by a bioinspired gyroid microstructure in solid state. ACS Nano 2018, 12, 485-493. [CrossRef] [PubMed]

4. Vu, D.T.; Chiu, H.W.; Nababan, R.; Le, Q.M.; Kuo, S.W.; Chau, L.K.; Ting, C.C.; Kan, H.C.; Hsu, C.C. Enhancing upconversion luminescence emission of rare earth nanophosphors in aqueous solution with thousands fold enhancement factor by low refractive index resonant waveguide grating. ACS Photonics 2018, 5, 3263-3271. [CrossRef]

5. Jiang, M.; Xie, H. Miscibility and morphology in block copolymer/homopolymer blends. Prog. Polym. Sci. 1991, 16, 977-1026. [CrossRef]

6. Zhao, J.Q.; Pearce, E.M.; Kwei, T.K. Binary and ternary blends of polystyrene-block-poly(p-hydroxystyrene). Macromolecules 1997, 30, 7119-7126. [CrossRef]

7. Han, Y.K.; Pearce, E.M.; Kwei, T.K. Poly(styrene-b-vinylphenyldimethylsilanol) and its blends with homopolymers. Macromolecules 2000, 33, 1321-1329. [CrossRef]

8. Kosoneen, H.; Ruokolainen, J.; Nyholm, P.; Ikkala, O. Self-organized cross-linked phenolic thermosets: Thermal and dynamic mechanical properties of novolac/block copolymer blends. Polymer 2001, 42, 9481-9486. [CrossRef] 
9. Dobrosielska, K.; Wakao, S.; Takano, A.; Matsushita, Y. Nanophase-separated structures of AB block copolymer/C homopolymer blends with complementary hydrogen-bonding interactions. Macromolecules 2008, 41, 7695-7698. [CrossRef]

10. Dobrosielska, K.; Wakao, S.; Suzuki, J.; Noda, K.; Takano, A.; Matsushita, Y. Effect of homopolymer molecular weight on nanophase-separated structures of $\mathrm{AB}$ block copolymer/C homopolymer blends with hydrogen-bonding interactions. Macromolecules 2009, 42, 7098-7102. [CrossRef]

11. Chen, S.C.; Kuo, S.W.; Jeng, U.S.; Su, C.J.; Chang, F.C. On modulating the phase behavior of block copolymer/homopolymer blends via hydrogen bonding. Macromolecules 2010, 43, 1083-1092. [CrossRef]

12. Dehghan, A.; Shi, A.C. Modeling hydrogen bonding in diblock copolymer/homopolymer blends. Macromolecules 2013, 46, 5796-5805. [CrossRef]

13. Tsai, S.C.; Lin, Y.C.; Lin, E.L.; Chiang, Y.W.; Kuo, S.W. Hydrogen bonding strength effect on self-assembly supramolecular structures of diblock copolymer/homopolymer blends. Polym. Chem. 2016, 7, 2395-2409. [CrossRef]

14. Hameed, N.; Guo, Q. Nanostructure and hydrogen bonding in interpolyelectrolyte complexes of poly(E-caprolactone)-block-poly(2-vinyl pyridine) and poly(acrylic acid). Polymer 2008, 49, 5268-5275. [CrossRef]

15. Hameed, N.; Liu, J.; Guo, Q. Self-assembled complexes of poly(4-vinylphenol) and poly( $\varepsilon$-caprolactone)-block-poly (2-vinylpyridine) via competitive hydrogen bonding. Macromolecules 2008, 41, 7596-7605. [CrossRef]

16. Hameed, N.; Guo, Q. Selective hydrogen bonding and hierarchical nanostructures in poly(hydroxyether of bisphenol A)/poly( $\varepsilon$-caprolactone)-block-poly(2-vinyl pyridine) blends. Polymer 2008, 49, 922-933. [CrossRef]

17. Chen, W.C.; Kuo, S.W.; Lu, C.H.; Jeng, U.S.; Chang, F.C. Self-assembly structures through competitive interactions of crystalline-amorphous diblock copolymer/homopolymer blends: Poly( $\varepsilon$-caprolactone-b-4-vinyl pyridine)/poly(vinyl phenol). Macromolecules 2009, 42, 3580-3590. [CrossRef]

18. Salim, N.V.; Hanley, T.; Guo, Q. Microphase separation through competitive hydrogen bonding in double crystalline diblock copolymer/homopolymer blends. Macromolecules 2010, 43, 7695-7704. [CrossRef]

19. Li, J.G.; Lin, Y.D.; Kuo, S.W. From microphase separation to self-organized mesoporous phenolic resin through competitive hydrogen bonding with double-crystalline diblock copolymers of poly(ethylene oxide-b-e-caprolactone). Macromolecules 2011, 44, 9295-9309. [CrossRef]

20. Salim, N.V.; Hameed, N.; Guo, Q. Competitive hydrogen bonding and self-assembly in poly(2-vinyl pyridine)-block-poly(methyl methacrylate)/poly(hydroxyether of bisphenol A) blends. J. Polym. Sci. Part B Polym. Phys. 2009, 47, 1894-1905. [CrossRef]

21. Hameed, N.; Salim, N.V.; Guo, Q. Microphase separation through competitive hydrogen bonding in self-assembled diblock copolymer/homopolymer complexes. J. Chem. Phys. 2009, 131, 214905. [CrossRef] [PubMed]

22. Lee, H.F.; Kuo, S.W.; Huang, C.F.; Lu, J.S.; Chan, S.C.; Wang, C.F.; Chang, F.C. Hydrogen-bonding interactions mediate the phase behavior of an $\mathrm{AB} / \mathrm{C}$ block copolymer/homopolymer blend comprising poly (methyl methacrylate-b-vinylpyrrolidone) and poly (vinylphenol). Macromolecules 2006, 39, 5458-5465. [CrossRef]

23. Chen, W.C.; Kuo, S.W.; Jeng, U.S.; Chang, F.C. Self-assembly through competitive interactions of miscible diblock copolymer/homopolymer blends: Poly (vinylphenol-b-methyl methacrylate)/poly (vinylpyrrolidone) blend. Macromolecules 2008, 41, 1401-1410. [CrossRef]

24. Zhou, J.; Shi, A.C. Microphase separation induced by differential interactions in diblock copolymer/homopolymer blends. J. Chem. Phys. 2009, 130, 234904. [CrossRef] [PubMed]

25. Lin, I.; Kuo, S.W.; Chang, F.C. Self-Assembly structures through competitive interactions of miscible crystalline-amorphous diblock copolymer/homopolymer blends. Polymer 2009, 50, 5276-5287. [CrossRef]

26. Matsushita, Y. Creation of hierarchically ordered nanophase structures in block polymers having various competing interactions. Macromolecules 2007, 40, 771-776. [CrossRef]

27. Miyase, H.; Asai, Y.; Takano, A.; Matsushita, Y. Kaleidoscopic tiling patterns with large unit cells from ABC star-shaped terpolymer/diblock copolymer blends with hydrogen bonding interaction. Macromolecules 2017, 50, 979-986. [CrossRef]

28. Asari, T.; Matsuo, S.; Takano, A.; Matsushita, Y. Three-phase hierarchical structures from AB/CD diblock copolymer blends with complemental hydrogen bonding interaction. Macromolecules 2005, 38, 8811-8815. [CrossRef] 
29. Asari, T.; Arai, S.; Takano, A.; Matsushita, Y. Archimedean tiling structures from ABA/CD block copolymer blends having intermolecular association with hydrogen bonding. Macromolecules 2006, 39, 2232-2237. [CrossRef]

30. Kuo, S.W. Hydrogen bond-mediated self-assembly and supramolecular structures of diblock copolymer mixtures. Polym. Int. 2009, 58, 455-464. [CrossRef]

31. Chen, W.C. , Kuo, S.W., Chang, F.C. Self-assembly of an A-B diblock copolymer blended with a C homopolymer and a C-D diblock copolymer through hydrogen bonding interaction. Polymer 2010, 51, 4176-4184. [CrossRef]

32. Kuo, S.W.; Tung, P.H.; Lai, C.L.; Jeong, K.U.; Chang, F.C. Supramolecular micellization of diblock copolymer mixtures mediated by hydrogen bonding for the observation of separated coil and chain aggregation in common solvents. Macromol. Rapid Commun. 2008, 29, 229-233. [CrossRef]

33. Kuo, S.W.; Tung, P.H.; Chang, F.C. Hydrogen bond mediated supramolecular micellization of diblock copolymer mixture in common solvents. Eur. Polym. J. 2009, 45, 1924-1935. [CrossRef]

34. Hsu, C.H.; Kuo, S.W.; Chen, J.K.; Ko, F.H.; Liao, C.S.; Chang, F.C. Self-assembly behavior of AB diblock and $\mathrm{CD}$ random copolymer mixtures in the solution state through mediated hydrogen bonding. Langmuir 2008, 24, 7727-7734. [CrossRef] [PubMed]

35. Tang, C.; Lennon, E.M.; Fredrickson, G.H.; Kramer, E.J.; Hawker, C.J. Evolution of block copolymer lithography to highly ordered square arrays. Science 2008, 322, 429-432. [CrossRef] [PubMed]

36. Tsai, C.C.; Gan, Z.; Chen, T.; Kuo, S.W. Competitive Hydrogen Bonding Interactions Influence the Secondary and Hierarchical Self-Assembled Structures of Polypeptide-Based Triblock Copolymers. Macromolecules 2108, 51, 3017-3029. [CrossRef]

37. Liu, C.C.; Chu, W.C.; Li, J.G.; Kuo, S.W. Mediated Competitive Hydrogen Bonding Form Mesoporous Phenolic Resins Templated by Poly (ethylene oxide-b- $\varepsilon$-caprolactone-b-L-lactide) Triblock Copolymers. Macromolecules 2014, 47, 6389-6400. [CrossRef]

38. Tsai, C.C.; Gan, Z.; Kuo, S.W. Functional Porous Polypeptide with Benzoxazine Chemistry from Bio-based Triblock Copolymer for Efficient Dye Adsorption. Polym. Chem. 2108, 9, 3684-3693. [CrossRef]

39. Tseng, T.C.; Kuo, S.W. Hydrogen-Bonding Strength Influences Hierarchical Self-Assembled Structures in Unusual Miscible/Immiscible Diblock Copolymer Blends. Macromolecules 2018, 51, 6451-6459. [CrossRef]

40. Tung, P.H.; Kuo, S.W.; Chen, S.C.; Lin, C.L.; Chang, F.C. Micellar Morphologies of Self-Associated Diblock Copolymers in Acetone Solution. Polymer 2007, 48, 3192-3200. [CrossRef]

41. Kuo, S.W.; Lin, C.L.; Chang, F.C. Phase Behavior and Hydrogen Bonding in Ternary Polymer Blends of Phenolic Resin/Poly(ethylene oxide)/Poly( $\varepsilon$-caprolactone). Macromolecules 2002, 35, 278-285. [CrossRef]

42. Kuo, S.W. Hydrogen-bonding in polymer blends. J. Polym. Res. 2008, 15, 459-486. [CrossRef]

43. Kuo, S.W. Hydrogen Bonding in Polymeric Materials; John Wiley \& Sons: Hoboken, NJ, USA, 2018.

44. Coleman, M.M.; Painter, P.C. Miscible Polymer Blends: Background and Guide for Calculations and Design; DEStech Publication Inc.: Lancaster, PA, USA, 2006.

45. Kuo, S.W.; Tung, P.H.; Chang, F.C. Syntheses and the study of strongly hydrogen-bonded poly (vinylphenol-b-vinylpyridine) diblock copolymer through anionic polymerization. Macromolecules 2006, 39, 9388-9395. [CrossRef]

46. Yeh, C.L.; Hou, T.; Chen, H.L.; Yeh, L.Y.; Chiu, F.C.; Muller, A.J.; Hadjichristidis, N. Lower Critical Ordering Transition of Poly(ethyleneoxide)-block-poly(2-vinylpyridine). Macromolecules 2011, 44, 440-443. [CrossRef]

47. Kwei, T.K. The Effect of Hydrogen Bonding on the Glass Transition Temperatures of Polymer Mixtures. J. Polym. Sci. Polym. Lett. Ed. 1984, 22, 307-313. [CrossRef]

Sample Availability: Samples of the compounds of PEO- $b$-P4VP and PS- $b$-PVPh are available from the authors. 